\title{
Gaze Tracking in Semi-Autonomous Grasping
}

\author{
Claudio Castellini \\ LIRA-Lab, University of Genova, Italy
}

\begin{abstract}
In critical human/robotic interactions such as, e.g., teleoperation by a disabled master or with insufficient bandwidth, it is highly desirable to have semi-autonomous robotic artifacts interact with a human being. Semi-autonomous grasping, for instance, consists of having a smart slave able to guess the master's intentions and initiating a grasping sequence whenever the master wants to grasp an object in the slave's workspace. In this paper we investigate the possibility of building such an intelligent robotic artifact by training a machine learning system on data gathered from several human subjects while trying to grasp objects in a teleoperation setup. In particular, we investigate the usefulness of gaze tracking in such a scenario. The resulting system must be light enough to be usable on-line and flexible enough to adapt to different masters, e.g., elderly and/or slow. The outcome of the experiment is that such a system, based upon Support Vector Machines, meets all the requirements, being $(a)$ highly accurate, $(b)$ compact and fast, and (c) largely unaffected by the subjects' diversity. It is also clearly shown that gaze tracking significantly improves both the accuracy and compactness of the obtained models, if compared with the use of the hand position alone. The system can be trained with something like 3.5 minutes of human data in the worst case.
\end{abstract}

Keywords: machine learning, gaze tracking, teleoperation

\section{Introduction}

Semi-autonomous teleoperation consists of having a robotic setup (the slave) work in a remote environment, guided by a human user (the master). In a basic setting, the cooperation between master and slave is realised in such a way that the actions performed by the master can be precisely and timely replicated by the slave. In order to convey a feeling of telepresence, in particular, a high bandwidth is required for the slave-to-master sensorial feedback (Batsomboon, Tosunoglu, \& Repperger, 2000). But, when any of the above conditions fails, teleoperation must be somehow augmented. The slave could consist of a set of surgical tools (Okamura, 2004) not immediately related to the human fingers; or, the master could be a disabled person; or, lastly, the master/slave communication bandwidth could be insufficient for a timely and accurate transmission of sensorial feedback.

One of the possibilities to overcome these problems is that of making the slave more intelligent by building into it internal models of the required actions (Kawato, 1999). It is envisioned, in this scenario, that the master

The work is supported by the EU-funded project NEURObotics (FP6-IST-001917). We thank Giulio Sandini and Giorgio Metta of the Italian Institute of Technology and Francesco Orabona of IDIAP for their support and suggestions. should first train the slave to perform the actions required, in a safe and controlled environment; and that this acquired knowledge should then be used by the slave in real situations, whenever the environment or the master's abilities do not allow direct control. Upon detecting the master's intention to, e.g., grasp an object in its own workspace, the slave should take control over, initiate and complete a grasping action possibly modelled upon the user's style, and then return the control to the master. This is what we call semiautonomous grasping.

As a minimum set of requirements, such a model should be accurate (it should be able to tell exactly when to start an autonomous grasp, avoiding doing it when not required), fast, since it must be used in real-time, and flexible: it must adapt well to different subjects' parameters (speed of reach, direction of motion), abilities and intentions, and it must be trained in a reasonably short amount of time.

In this paper we investigate the possibility of building such an internal model, employing a machine learning system based upon a Support Vector Machine (SVM) (Boser, Guyon, \& Vapnik, 1992). In (Castellini \& Sandini, 2007), we already showed some promising results related to the problem; here we refine the data analysis and show the results of an experiment in which seven subjects, of different ages and with slightly different movement and gaze abilities, were placed in a real teleoperation scenario; by repeatedly performing a fake grasping action, the subjects would 
teach a SVM to recognise when they wanted to grasp an object in the slave setup; this was achieved by simply fixating the object, reaching for it and closing their hand. Here we carry the data analysis to the end.

Data was gathered from the subjects using a magnetic tracker for the position of the hand, and a gaze tracker in order to understand whether the subject was fixating a particular object. One interesting question is whether gaze tracking couldactually improve the situation. The outcome of the experiment is that such a model can actually be built, and that it fulfills all the requirements enumerated above: it is highly accurate; the solution achieved is extremely compact; and these characteristics are largely independent of the subjects' abilities. The training phase is accomplished using about 3.5 minutes of data gathered in real-time from each subject, in the worst case. Lastly, the use of gaze tracking significantly improves the obtained models, both as far as their accuracy and size is concerned.

The paper is structured as follows: we first describe the materials and methods used in the experiment; we then go on to show the experimental results, and lastly we draw conclusions and outline future work.

\section{Materials and Methods}

\section{Subjects}

Seven subjects, four women and three men, aged 30 to 73 , volunteered to join the experiment. They were all right-handed and fully able-bodied, and were given no knowledge of the aim of the experiment. Four of the subjects were slightly visually impaired.

\section{Setup and devices}

The subjects were asked to sit confortably in front of a clean workspace, and a flat 17 inches color monitor was placed in front of them at a distance of about half a meter. They wore an Immersion CyberGlove data glove (Virtual Technologies, Inc., 1998) on their right hand, and an Ascension Flock-of-Birds (FoB) (Ascension Technology Corporation, 1999) sensor was firmly mounted on top of their wrist. Lastly, an ASL E504 gaze tracker (Applied Science Laboratories, 2001) was placed on the left hand side of the monitor. Figure 1 shows the devices and setup.

The FoB had the $X / Y$ plane parallel to the workspace horizontal plane. The device returns 6 doubleprecision numbers describing the position $(x, y$ and $z$ in inches) and rotation (azimuth, elevation and roll in degrees) of the sensor with respect to a magnetic basis mounted about one meter away from the subject. The spatial resolution of the acquired data is 0.1 inches and 0.5 degrees.

The E504, after the standard calibration phase, returns one true/false value, denoting validity of the gaze coordinates (that is, the pupil being in sight of the camera and correctly recognised), and two doubleprecision numbers indicating the coordinates of the subject's gaze with respect to the monitor. Its precision is about 1 degree which, we verified, corresponded to less than one pixel precision on the monitor, which was considered acceptable. The device can nominally stream at up to $50 \mathrm{~Hz}$. We configured it in order not to filter in any way the gaze signal, so that we could read the "raw" pupil movement; notice that, however, the signal was actually filtered off-line, later on, during the data analysis, as described below.

The CyberGlove was used as an on-off switch, to detect when the subject's hand would close, by monitoring one of its sensors via a threshold.

The monitor showed the slave's workspace; the slave is the humanoid platform Babybot, composed of two colour cameras, a commercial 6-degrees-offreedom robotic arm, a pan/tilt head and a humanoid hand (see, e.g., (Natale, Orabona, Berton, Metta, \& Sandini, 2005)). During the experiment, we only employed one of its colour cameras. Figure 1, panel $(d)$ (reproduced from (Natale et al., 2005)), shows a blackand-white representation of the workspace as seen on the monitor by the subject, that is, the "stimulus" presented to those who joined the experiment.

All data were collected, synchronised, and saved in real time at a frequency of slightly less than $50 \mathrm{~Hz}$, this being the best frequency obtained from the gaze tracker.

\section{Method}

The subjects were asked to initially keep their right hand and arm in a resting position. The monitor showed the slave's workspace, in which several objects could be clearly seen, and a moving red cross corresponding to the detected subject's gaze. The subjects were then instructed, upon a request by the experimenter, to look at one of the objects on the monitor and then to move their hand as if to reach and grasp it, signalling the act of grasping by closing their right hand. The red cross on the screen turned green when the hand was closed, to confirm the grasping.

This fake grasping act was repeated for 15 to 21 times, each time with a different object (therefore, toward a different position) seen on the monitor. The maximum duration of the whole experiment for a single subject was about 3.5 minutes, resulting in no tiredness.

\section{Building the data set}

The first question was what pieces of data to consider to train our machine, that is, how to filter and/or manipulate the data obtained from the setup. It is wellknown that, when a human subject wants to grasp an object, he/she fixates the desired object and then performs the reaching action, without looking at his own hand while reaching (see, e.g., (Johansson, Westling, 


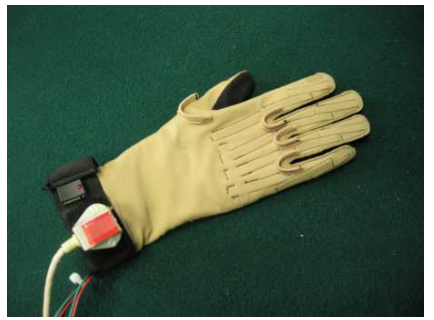

(a)

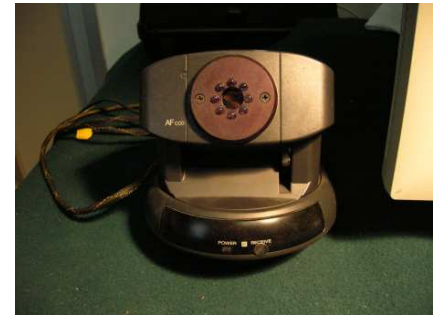

(b)

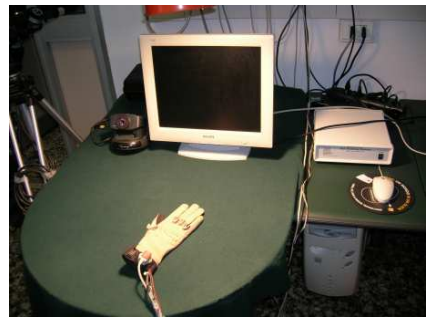

(c)

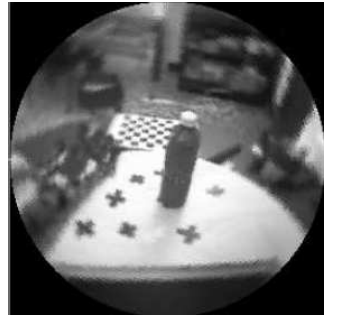

(d)

Figure 1. The devices and setup used for the experiment, and the stimulus presented to the human subjects: $(a)$ the Immersion CyberGlove with the Flock-of-Birds sensor just above the wrist; $(b)$ the ASL E504 gaze tracker (pan/tilt near-infrared camera); $(c)$ the whole setup; $(d)$ a black-and-white image of the stimulus, that is, an example of what a subject might see in the monitor.

Bäckström, \& Flanagan, 2001)). Therefore, we considered $(a)$ the average of the subjects' hand velocity, $(b)$ the variance of the subjects' gaze coordinates and $(c)$ the information whether the subjects' right hand was open or closed. We then expect, while fixating and reaching,

1. the gaze coordinates to hover around the point on the screen where the desired object is seen; that is, their standard deviation over some time to be small; and

2. the hand to move towards the object on the screen, that is, the hand velocity components to be on average large.

The instants in which the hand is closed signal the intention to grasp, whereas those when the hand is open represent negative examples. Data $(a)$ were easily obtained by differentiating in time the hand position $x, y, z$ coordinates obtained from the FoB and then averaging these numbers over a certain time window (see below); data $(b)$ were obtained by evaluating the standard deviation over the same time window of the gaze coordinates obtained from the E504; and lastly data $(c)$ was obtained directly from the CyberGlove. (The samples corresponding to negative values of the E504 validity flag were ignored, manually verifying that this would not hamper the overall statistics.)

Thus, from each subject we obtained a sequence of 6-tuples (the three hand velocity coordinates, the two gaze coordinates and the open/closed hand flag). The above considerations should be valid over a certain time window, characteristic of the fixation/reaching operations - call it $\tau$; and in general each subject will have a different $\tau(i), i=1, \ldots, 7$. Driven by this, we then decided to feed the learning system the following data: for each user $i$ (and therefore for each sequence) and for a range of different values $T_{c}$ attributed to $\tau(i)$, the hand velocity average values over $T_{c}$ (three real numbers) and the gaze position standard deviations over $T_{c}$ (two real numbers). Training was enforced by requiring that the system could guess, instant by instant, whether the hand was closed or not. This was represented as an integer value, in turn 1 or -1 . The problem of guessing when the subject wants to grasp was thus turned into a typical supervised learning problem.

\section{Grasping speed}

In choosing the range for $T_{c}$, we were driven by the main consideration that a moving time window should not be longer then the interval of time between one grasping attempt and the following one. In fact, a longer time window could trick the system into considering data obtained during two or more independent grasping attempts.

By examining all sequences we found out that the interval between one grasping attempt and the following one lasted on average $7.1 \pm 1.8$ seconds. We then decided to let $T_{c}$ range in the interval $0.1, \ldots, 5$ seconds.

In general, we expected to find a best minimum value for $T_{c}$, which would then be the required $\tau(i)$ for each user, figuring out that shorter values would convey too little information about the ongoing movement, and that for longer ones, the moving averages would reach a plateau effect, tending to the overall average values of the hand velocity and gaze standard deviations. In fact, a moving average is roughly equivalent to a low-pass filter, and the longer the $T_{c}$, the lower the cutoff frequency; evaluating the best minimum value for $T_{c}$ is tantamount to finding the right cutoff frequency, that is, to filtering out noise without damaging the signal.

\section{Support Vector Machines}

Our machine learning system is based upon Support Vector Machines (SVMs). Introduced in the early 90s by Boser, Guyon and Vapnik (Boser et al., 1992), SVMs are a class of kernel-based learning algorithms deeply rooted in Statistical Learning Theory (Vapnik, 1998), now extensively used in, e.g., speech recognition, object classification and function approximation with good results (Cristianini \& Shawe-Taylor, 2000). For an extensive introduction to the subject, see, e.g., (Burges, 1998).

We are interested here in the problem of SVM classification, that is: given a set of $l$ training samples $S=\left\{\mathbf{x}_{i}, y_{i}\right\}_{i=1}^{l}$, with $\mathbf{x}_{i} \in \mathbb{R}^{m}$ and $y_{i} \in\{-1,1\}$, find a function $f$, drawn from a suitable functional space $\mathcal{F}$, which best approximates the probability distribution of the 
source of the elements of $S$. This function will be called a model of the unknown probability distribution. In order to decide whether a sample belongs to either category, the sign of $f$ is considered, with the convention that $\operatorname{sgn}(f(\mathbf{x})) \geq 0$ indicates $y=1$ and vice-versa. In practice, $f(\mathbf{x})$ is a sum of $l$ elementary functions $K(\mathbf{x}, \mathbf{y})$, each one centered on a point in $S$, and weighted by real coefficients $\alpha_{i}$ :

$$
f(\mathbf{x})=\sum_{i=1}^{l} \alpha_{i} K\left(\mathbf{x}, \mathbf{x}_{i}\right)+b
$$

where $b \in \mathbb{R}$. The choice of $K$, the so-called kernel, is done a priori and defines $\mathcal{F}$ once and for all; it is therefore crucial. According to a standard practice (see, e.g., (Cristianini \& Shawe-Taylor, 2000)) we have chosen a Gaussian kernel, which has one positive parameter $\sigma \in \mathbb{R}$ which is the standard deviation of the Gaussian functions used to build (1). Notice that this is not related to the fact that the target probability distribution might or might not be Gaussian.

Now, let $C \in \mathbb{R}$ be a positive parameter; then the $\alpha_{i} \mathrm{~s}$ and $b$ are found by minimising $L_{P}$ (training phase) with respect to the coefficients $\alpha_{i}$, where

$$
L_{P}=R(S, K, \alpha)+C \sum_{i=1}^{l} L\left(\mathbf{x}_{i}, y_{i}, f\right)
$$

Here $R$ is a regularisation term and $L$ is a loss functional. In practice, after the training phase, some of the $\alpha_{i}$ s will be zero; the $\mathbf{x}_{i}$ s associated with non-zero $\alpha_{i}$ s are called support vectors. Both the training time (i.e., the time required by the training phase) and the testing time (i.e., the time required to find the value of a point not in $S$ ) crucially depend on the total number of support vectors; therefore, this number is an indicator of how hard the problem is. Since the number of support vectors is proportional to the sample set (Steinwart, 2003), an even better indicator of the hardness of the problem is the percentage of support vectors with respect to the sample set size. We will denote this percentage by the symbol $p_{S V}$ and call it size of the related model. Willing to implement the system on-line, one has to choose models with the smallest possible size.

In (2), minimising the sum of $R$ and $L$ together ensures that the solution will approximate well the values in the training set, at the same time avoiding overfitting, i.e., exhibiting poor accuracy on points outside $S$. Smaller values of the parameter $C$ give more importance to the regularisation term and vice-versa.

There are, therefore, two parameters to be tuned in our setting: $C$ and $\sigma$. In all our tests we found the optimal values of $C$ and $\sigma$ by grid search with 3 -fold crossvalidation. This ensures that the obtained models will have a high generalisation power, i.e., their guess will be accurate also on samples not in $S$.

Notice, lastly, that the quantity to be minimised in Equation (2) is convex; due to this, as well as to the use of a kernel, SVMs have the advantages that their training is guaranteed to end up in a global solution and that they can easily work in highly dimensional, non-linear feature spaces, as opposed to analogous algorithms such as, e.g., artificial neural networks. As a matter of fact, SVMs are best employed when the chosen kernel maps the samples to a space in which the problem is linearly separable, that is, a hyperplane (linear function) can be found which separates the samples labelled 1 from those labelled -1 .

We have employed LIBSVM v2.82 (Chang \& Lin, 2001), a standard, efficient implementation of SVMs.

According to the procedure described in the previous parts of this Section, we decided to set up a SVM for each user $i$ and value of the time window $T_{c}$. Willing to compare the performance with and without the use of the gaze signal, we defined $\mathbb{R}^{3}$ as the input space in the case of not using the gaze (the 3 numbers representing the hand average velocity over $T_{c}$ ), and $\mathbb{R}^{5}$ in the case of using the gaze (the 5 numbers representing the hand velocity average and gaze position standard deviation over $T_{c}$ ). According to this and to the experience gathered in previous work (Castellini \& Sandini, 2007), the ranges of the parameters $C$ and $\sigma$ were chosen as follows: $C$ was $10^{k}$ with $k=-1, \ldots, 3$ in steps of 0.2 , whereas $\sigma$ was $\sqrt{\frac{5}{10^{k}}}$ with $k=0, \ldots, 2$, in steps of 0.2 $\left(\sqrt{\frac{3}{10^{k}}}\right.$ in the case of not using the gaze).

\section{Experimental Results}

In (Castellini \& Sandini, 2007), we already showed that SVMs clearly outperform a simple decision tree in solving the problem; so we will be using SVMs alone in this paper. Morover, it was therein noted that the standard measure of performance for a SVM classifier (that is, the fraction of correctly guessed labels over the total number of samples) is biased, at least in two ways: firstly, the number of 1 labels is much smaller than that of -1 labels; as a consequence, a dumb predictor which guessed -1 identically would achieve an average accuracy of about $83 \%$. Therefore we adopt a weigthed measure of accuracy, $\frac{n_{-1} c_{1}+n_{1} c_{-1}}{c_{1}+c_{-1}}$, where $n_{i}$ is the number of correctly guessed $i$ labels, $i \in\{1,-1\}$, and $c_{i}$ is the total number of $i$ labels.

Secondly, we are looking for both accurate and small models; this means that looking for the most accurate models could lead to unnecessarily large models. Therefore, we adopt here an index of performance given by the ratio of the weighted accuracy detailed above and the percentage of support vectors in the obtained model. This index was used for grid searching the best values of $C$ and $\sigma$.

Figure 2 shows the weighted accuracy and size of the obtained models as $T_{c}$ is increased, both when using and not using the gaze. 

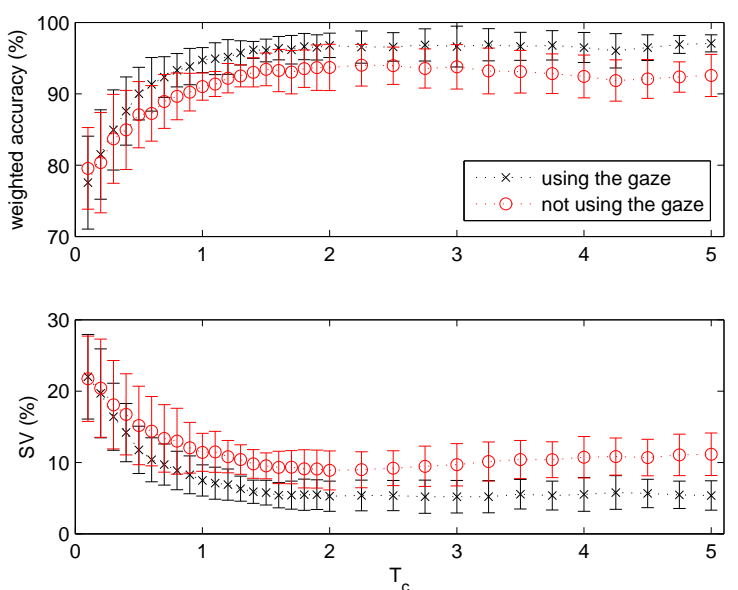

Figure 2. Comparison between not using the gaze (red curve) and using the gaze (black one) as $T_{c}$ increases. Top panel: weighted accuracy. Bottom panel: size of the models, expressed as the fraction of Support Vectors. Dots are the mean values over all subjects, whereas the errorbars denote \pm one standard deviation.

First of all, the effect predicted in the previous Section is present: both the accuracy and size of the models become better as $T_{c}$ is increased, and reach a maximum around $T_{c}=2$ seconds; then they remain essentially constant. Secondly, notice that the use of the gaze uniformly and consistently improves both the accuracy of the models and their size, the black curve being systematically higher than the red one, as far as the accuracy is concerned, and lower in the case of the model size. For $T_{c} \geq 2$, there is little overlap among the errorbars, indicating a stastically significant improvement. Thirdly, it seems that such a value of $T_{c}$ is just about right for all subjects, notwithstanding their differences; we then conclude that $\tau(i)$ is essentially the same for all subjects. This could dramatically reduce the setup time in a real setting.

For $T_{c}=2$ seconds, the accuracy is $96.79 \% \pm 1.7 \%$ using the gaze, as opposed to $93.69 \% \pm 3.24 \%$ when not using it; the model size is $5.27 \% \pm 2.12 \%$ as opposed to $8.88 \% \pm 2.73 \%$. The mean values are better, and the standard deviations are smaller when using the gaze, denoting better performance and higher robustness with respect to the diversity of the subjects.

Let us then turn to the experiment with the gaze, and analyse the performance in deeper detail for the single subjects. Figure 3 shows the same results as the black curves of Figure 2, but for each subject.

Consider the Figure, panel $(a)$ : it is apparent that the worst subject is number 5, a 73-years old woman who has undergone in the past a cataract surgical operation. It is not surprising that this is the hardest subject; still, for $T_{c}=2$, the model has a remarkable accuracy of $93.03 \%$. All other subjects reach an accuracy of $96.75 \%$ and more for the same value of $T_{c}$. Analogous consider- ations apply as far as the model size is concerned (panel (b) of the same Figure). It is interesting to note that, even for such a hard subject as subject 5 , the use of the gaze signal greatly improves the accuracy of the model (see Figure 4).

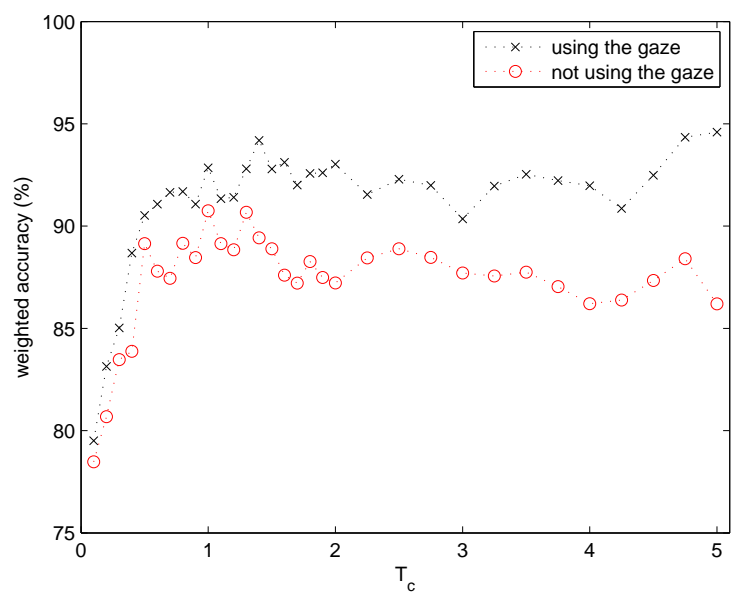

Figure 4. Comparison between not using the gaze (red curve) and using the gaze (black one) as $T_{c}$ increases, for subject 5 .

\section{Cross-subject analysis}

One further interesting point is: how well can these models be transferred across subjects? In other words: are models trained on a certain subject $i$ good for predicting the intention of another subject $j$ ? Does gaze improve the situation? And, what are the "hardest" subjects to be modelled? In order to answer these questions we have tested each model for $T_{c}=2$ on data gathered from all subjects, both when using and not using the gaze. Figure 5 shows the results as cross-accuracy matrices: in each matrix $A$, the entry $A_{i j}$ represents in colour the accuracy attained by the model trained on subject $i$ when tested on data gathered from subject $j$.

As is apparent, using the gaze improves the situation: the overall accuracy (not considering the diagonal elements of the matrices, of course) is $55.97 \% \pm$ $8.6 \%$ when not using the gaze, as opposed to $61.32 \% \pm$ $11.77 \%$ when using the gaze. In general these figures are not very good, meaning that there is little chance that models can be transferred across subjects, even when using the gaze.

Let us now restrict to the experiment with the gaze (right panel of the Figure). The worst model (row of the matrix) is, unsurprisingly, obtained from subject number 5, with an accuracy of $49.31 \% \pm 2.8 \%$; as well, the hardest subject (column of the matrix) is again number 5 , with an accuracy of $56.70 \% \pm 9.09 \%$. Subject 5 has to be treated on her own. But as well, again, let us remark that the two best models, obtained from subjects $2(73.99 \% \pm 7.89 \%)$ and $6(70.32 \% \pm 6.61 \%)$ still show 


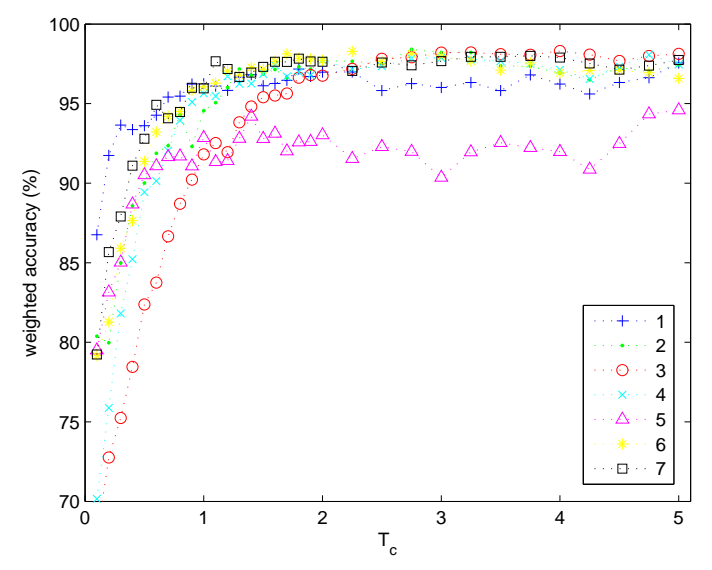

(a)

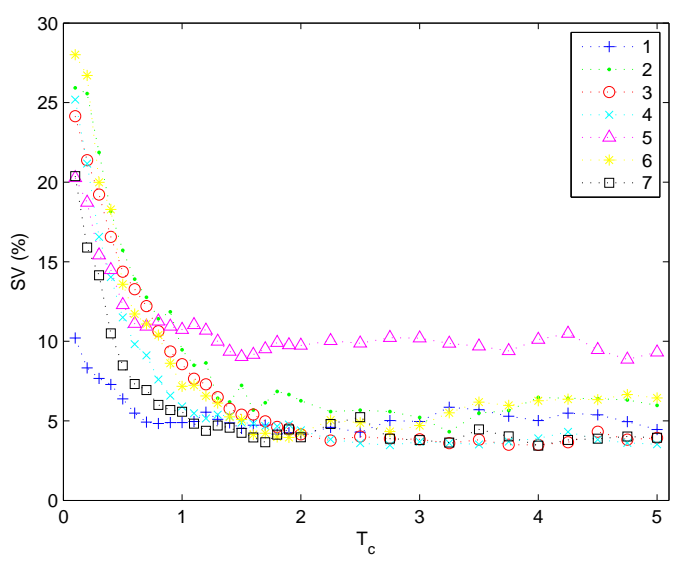

(b)

Figure 3. Accuracy $(a)$ and size $(b)$ of the models for each single subject, using the gaze.
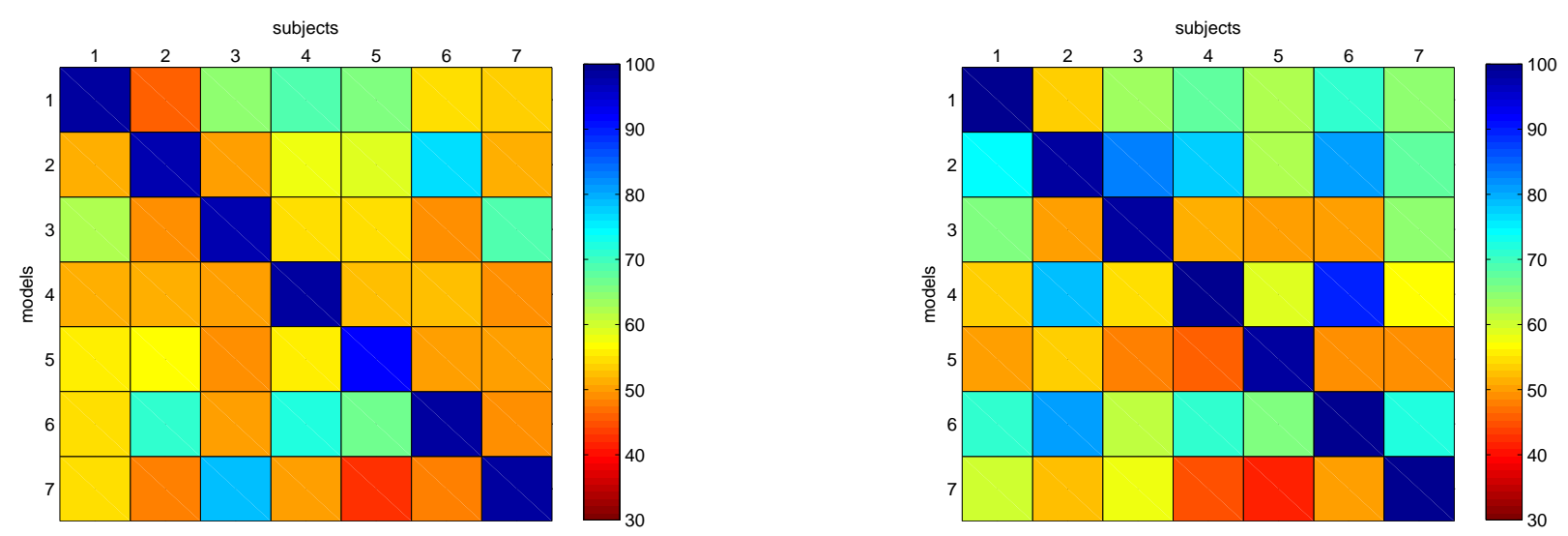

Figure 5. Cross-subject accuracy, not using the gaze (left panel, accuracy 55.97\% $\pm 8.6 \%$ ) and using the gaze (right panel, accuracy $61.32 \% \pm 11.77 \%)$.

a poor trasnferability as well - compare these figures with those of the previous Subsection.

\section{Discussion and Conclusions}

In this paper we have shown that good artificial internal models of when to grasp can be obtained by applying Support Vector Machines to data gathered from diverse human subjects, engaged in a simple grasping experiment in a teleoperation scenario. The data consisted of the hand velocity and the gaze signal, which was proved to be crucial in improving the performance of the models, both in terms of accuracy and size.

Interestingly, about the same characteristic time value for $T_{c}$ can be found for all subjects, the best models being found for $T_{c}=2$ seconds. The system greatly benefits from the use of gaze tracking, also in case the subjects are visually impaired (subject 5).

The models obtained for each subject are $(a)$ highly accurate, giving the correct guess in $96.79 \% \pm 1.7 \%$ of the cases; and (b) small, and therefore fast and usable in an on-line environment, the percentage of support vectors for each model being $5.27 \% \pm 2.12 \%$. Analogous figures when the gaze is not used are sensibly worse. The accuracy and size of the best models for each subject are remarkable, meaning that the system is also very flexible and unhindered by the subjects' diverse abilities and visual impairments.

Cross-subject transferability of models is, on the other hand, so far unfeasible, the figures showing that models trained on a subject cannot in general obtain good accuracy values when applied to data gathered from different subjects. But this does not seem of any hindrance to the approach, since the models can be trained, in the worst case, using 3.5 minutes of user data. Independent training for each subject can then be performed with a reasonable effort. 


\section{References}

Applied Science Laboratories. (2001). Eye tracking system instruction manual - model 504 pan/tilt optics - v2.4 [Computer software manual]. 175 Middlesex Turnpike, Bedford, MA 01730.

Ascension Technology Corporation. (1999, January). The flock of birds - installation and operation guide [Computer software manual]. PO Box 527, Burlington (VT), USA.

Batsomboon, P., Tosunoglu, S., \& Repperger, D. W. (2000). A survey of telesensation and teleoperation technology with virtual reality and force reflection capabilities. International Journal of Modeling and Simulation, 20(1), 79-88.

Boser, B. E., Guyon, I. M., \& Vapnik, V. N. (1992). A training algorithm for optimal margin classifiers. In D. Haussler (Ed.), Proceedings of the 5th annual acm workshop on computational learning theory (pp. 144-152). ACM press.

Burges, C. J. C. (1998). A tutorial on support vector machines for pattern recognition. Knowledge Discovery and Data Mining, 2(2).

Castellini, C., \& Sandini, G. (2007). Learning when to grasp. (Invited paper at Concept Learning for Embodied Agents, a workshop of ICRA 2007, Rome, Italy. Available at http://people.liralab.it/drwho/publications/ 2007. CLEA.pdf)

Chang, C.-C., \& Lin, C.-J. (2001). LIBSVM: a library for support vector machines [Computer software manual]. (Soft- ware available at http://www.csie.ntu.edu.tw/ cjlin/ libsvm)

Cristianini, N., \& Shawe-Taylor, J. (2000). An introduction to support vector machines (and other kernel-based learning methods). Cambridge University Press.

Johansson, R. S., Westling, G., Bäckström, A., \& Flanagan, J. R. (2001). Eye-hand coordination in object manipulation. J. Neurosci., 21, 6917-6932.

Kawato, M. (1999). Internal models for motor control and trajectory planning. Current Opinion in Neurobiology, 9, 718727.

Natale, L., Orabona, F., Berton, F., Metta, G., \& Sandini, G. (2005). From sensorimotor development to object perception. In Proceedings of the international conference on humanoid robotics.

Okamura, A. M. (2004). Methods for haptic feedback in teleoperated robot-assisted surgery. Industrial robot, 31(6), 499-508.

Steinwart, I. (2003). Sparseness of support vector machines. Journal of Machine Learning Research, 4, 1071-1105.

Vapnik, V. N. (1998). Statistical learning theory. New York: John Wiley and Sons.

Virtual Technologies, Inc. (1998, August). Cyberglove reference manual [Computer software manual]. 2175 Park Blvd., Palo Alto (CA), USA. 